\title{
Interprofessional clinical skills: a tool for developing clinical skills, team working and professional awareness between medical students and physician associates
}

\author{
Author: David Holland
}

\section{Introduction and aims}

Physician associates (PAs) perform a relatively new role in the medical team, with a similar skill set to a junior doctor (JD). Their role is locally yet to be clearly defined and it is anticipated they will assist clinical teams by performing many of the tasks that a JD would traditionally undertake.

At the University of East Anglia (UEA), the first cohort of PAs are about to graduate and take up clinical roles. They will work closely with JDs. Neither JDs nor PAs have much experience of working together locally.

Interprofessional clinical skills (ICS) training is known to improve clinical skills, communication skills and team working.

It also has the benefit of providing a contextual learning experience in which the roles, responsibilities and skill sets of other healthcare professionals can be better understood. This may enhance understanding of professional identity within the medical team, improve efficiency and patient safety when graduates start in clinical practice. The aim of this project was to develop an educational tool to prepare students of different backgrounds using interprofessional clinical skills.

\section{Methods}

A pilot objective structured clinical examination-style ICS session was designed with three stations of 20 -minutes duration. PAs ( $n=9$ ) and final-year medical students (MSs; $n=9$ ) were recruited and paired. Three cycles were undertaken. Each station had a tutor and feedback was given contemporaneously. Student feedback was obtained in a debriefing focus group and from a feedback form, in which parameters were measured from strongly disagree (1) to strongly agree (5).

\section{Results}

Feedback from both PAs and MSs was positive. Students enjoyed the sessions (mean 4.61) and worked effectively with their partner (mean 4.39). Students reported the session made them more confident in their clinical skills (mean 4.39), was a good way of learning interprofessional skills (mean 4.28) and was a good educational tool (mean 4.66).

Students found it taught them about the role of their partner (mean 3.89). This was stronger among MSs (mean 4.33) than PAs (mean 3.44). Students would like further teaching opportunities like this (mean 4.66) and the students reported they would recommend the session to friends (mean 4.72). The students found the session a non-stressful event (mean 2.56).

\section{Conclusion}

ICS between PAs and MSs provides an enjoyable, non-stressful, learning environment. Students feel it enhances their clinical skills, and also their team working and knowledge of the roles of allied healthcare professionals. This will help prepare the students for life as a medical professional.

\section{Conflict of interest statement}

No conflicts of interests were found. 